\title{
Adsorption studies of iron(III) on chitin
}

\author{
G KARTHIKEYAN*, N MUTHULAKSHMI ANDAL and K ANBALAGAN \\ Department of Chemistry, Gandhigram Rural Institute - Deemed University, Gandhigram 624 302, India \\ e-mail: drg_karthikeyan@rediffmail.com
}

MS received 18 December 2003; revised 4 June 2005

\begin{abstract}
Adsorption of ferric ions by chitin was studied by the batch equilibration method. The influence of particle size and dosage of the adsorbant, contact time, initial concentration of the adsorbate and temperature were experimentally verified. The effect of anions like chloride, nitrate and sulphate and also of cations like zinc, chromium and copper on the adsorption of iron(III) was determined. The time dependence of fraction of adsorption, $Y_{t}$, at varying particle sizes and doses of chitin and the intraparticle diffusion rate constants, $k_{p}$, of the adsorption process were calculated. Thermodynamic and equilibrium parameters of the reaction were determined to understand the sorption behaviour of chitin. The results revealed that the adsorption of iron(III) by chitin is spontaneous, endothermic and favourable.
\end{abstract}

Keywords. Adsorption; chitin; variable parameters; fraction of adsorption; temperature effect.

\section{Introduction}

Iron is one of the major constituents of the lithosphere and comprises approximately $5 \%$ of it. It is routinely detected in municipal waste effluent, particularly in cities where iron and steel are manufactured. Iron readily complexes with sulphates in the sediments of many surface waters. The primary concern about the presence of iron in drinking water is its objectionable taste. The taste of iron in drinking water can be easily detected even at low concentrations of about $1.8 \mathrm{mg} / \mathrm{l}^{1}{ }^{1}$ There are many problems that result from iron toxicity. These include anorexia, oligura, diarrhoea, hypothermia, diphasic shock, metabolic acidosis and even death. In addition to these, the patient may experience vascular congestion of the gastrointestinal tract, liver, kidneys, heart, brain, spleen, adrenals and thymus. With acute iron poisoning, much of the damage to the gastrointestinal tract and liver may result from the high localized iron concentration and free radical production leading to heptatoxicity via lipid peroxidation and destruction of the hepatic mitochondria. As a result of iron storage disease, the liver becomes cirrhotic. Hepatoma, a primary cancer of the liver, has become the most common cause of death among patients with hemochromatosis. ${ }^{2}$ This is an iron storage disease that results from the inability of the intestine to keep out unwanted iron. Instead,

*For correspondence this iron accumulates in the liver causing siderosis ${ }^{3}$ (accumulation of storage iron in tissues) and causes damage to the storage organs. Also, when siderosis becomes severe in young people, it leads to myocardial disease which is a common cause of death. Impotence may also occur in young men and amenorrhea in young women. Both these problems relating to reproduction are due to iron loading in the anterior pituitary.

This study deals with the removal of ferric ions from aqueous systems using chitin as an adsorbent. Chitin is a polysaccharide and is a major component of arthropod and crustacean shells, like those of lobsters, shrimps or crabs. Chitin is a non-toxic and biodegradable polymer of $\mathrm{N}$-acetylglucosamine and glucosamine residues. ${ }^{4}$ The constituent monosaccharide units in chitin are linked together by $\beta-1,4$-glycosidic bonds and hence have a relatively rigid configuration. It is easily filmable and spinnable and the materials thus processed have very good mechanical properties. It is insoluble in water and alkali but soluble in strong mineral acids and in anhydrous formic acid. It forms complexes with transition metal ions. A systematic study of the adsorption of ferric ion by chitin under different experimental conditions has been carried out to understand the nature of the reaction.

\section{Experimental}

Commercially available chitin (Otto Kemi, C1904) was obtained in the form of flakes from M/s BR 
Corporation, Mumbai. The raw sample was pulverized using mortar and pestle and sieved into three sizes, viz., $0 \cdot 21,0.50$ and $0.71 \mathrm{~mm}$, using scientific test molecular sieves manufactured by Elite Scientific Instruments Co., Mumbai. Chitin samples of definite particle sizes were further purified by first boiling in $0 \cdot 1 \mathrm{~N} \mathrm{HCl}$ for three hours. The solution was decanted and the residue was treated further by boiling in $0 \cdot 1$ $\mathrm{N} \mathrm{NaOH}$ for another three hours. The purified samples were washed several times and were soaked in double distilled water till neutral $\mathrm{pH}$. Soaking of chitin resulted in swelling which results in more reaction sites in the adsorbent material. Later the samples were dried in an oven. Only the chemically treated samples were used for the adsorption experiments. All experimental solutions were prepared using only double distilled water. The adsorption experiments were carried out by batch equilibration method. All the chemicals employed for the analysis were AnalaR grade. A stock solution of the adsorbate containing $1000 \mathrm{mg} / \mathrm{l}$ of iron(III) ions was prepared using ammonium ferric sulphate $\left[\mathrm{NH}_{4} \mathrm{Fe}\left(\mathrm{SO}_{4}\right)_{2} \cdot 12 \mathrm{H}_{2} \mathrm{O}\right]$. From the stock solution, aliquots containing different concentrations viz., $2,4,6,8,10,12$ and $14 \mathrm{mg} / \mathrm{l}$ of iron(III) ions were measured out into separate iodine flasks. A sample adsorbate of each concentration $(50 \mathrm{ml})$ was mixed with $10 \mathrm{mg}$ of chitin and the mixtures were agitated in a mechanical shaker. Adsorption experiments with chitin samples of particle sizes $0.21,0.50$ and $0.71 \mathrm{~mm}$ were separately carried out. The solutions were filtered at regular intervals of 2 minutes using Whatman No. 41 filter paper and the concentration of iron(III) ions in the filtrate at each stage was determined using an atomic absorption spectrometer (Model AA 100, Perkin-Elmer). Adsorption experiments were conducted at $\mathrm{pH} 3$. This was because of the formation of ferric hydroxide at higher $\mathrm{pH}$ range with sodium hydroxide which was added to adjust the $\mathrm{pH}$ of the acidic ferric solution. The experiments were repeated at four different temperatures viz., 293, 303, 313 and $323 \mathrm{~K}$ to determine the effect of temperature on the sorptive behaviour of chitin. All experiments were done in a thermostat with a shaker attached (Techno) which maintained temperature within $\pm 0 \cdot 1^{\circ} \mathrm{C}$. Adsorption studies of iron(III) ions by chitin were repeated in the presence of copper, zinc and chromium ions to examine the influence of co-ions on the sorptive nature of the adsorbent. The concentrations of residual iron(III) ions from the mixture containing equal amounts of copper, zinc and chromium ions were determined. Duplicate measurements were made such that the residual concentration values were reproducible within $\pm 2 \%$.

\section{Results and discussion}

\subsection{Effect of particle size}

Preliminary adsorption studies of metal ions by chitin indicated that sorption capacity is dependent on its particle size. Figure 1 represents the uptake of iron(III) by chitin of three different particle sizes, viz., 0.21, 0.50 and $0.71 \mathrm{~mm}$. The sample with particle size $0.21 \mathrm{~mm}$ exhibits maximum adsorption efficiency and this is attributed to the larger surface area of the adsorbent material. Diffusional resistance to mass transport in the case of adsorbent with larger particle sizes is higher and most of the internal surface of these particles may not be utilized for adsorption. Consequently, the amount of metal ions adsorbed is less in such cases. ${ }^{5}$ Since the extent of adsorption is considerably higher in the case of chitin samples of $0.21 \mathrm{~mm}$ particle sizes, it was decided to limit the discussions of the adsorption experiments to that with chitin of $0.21 \mathrm{~mm}$ particle size.

\subsection{Effect of contact time}

Contact time required for the maximum removal of iron(III) by chitin is represented by figure 2 . There

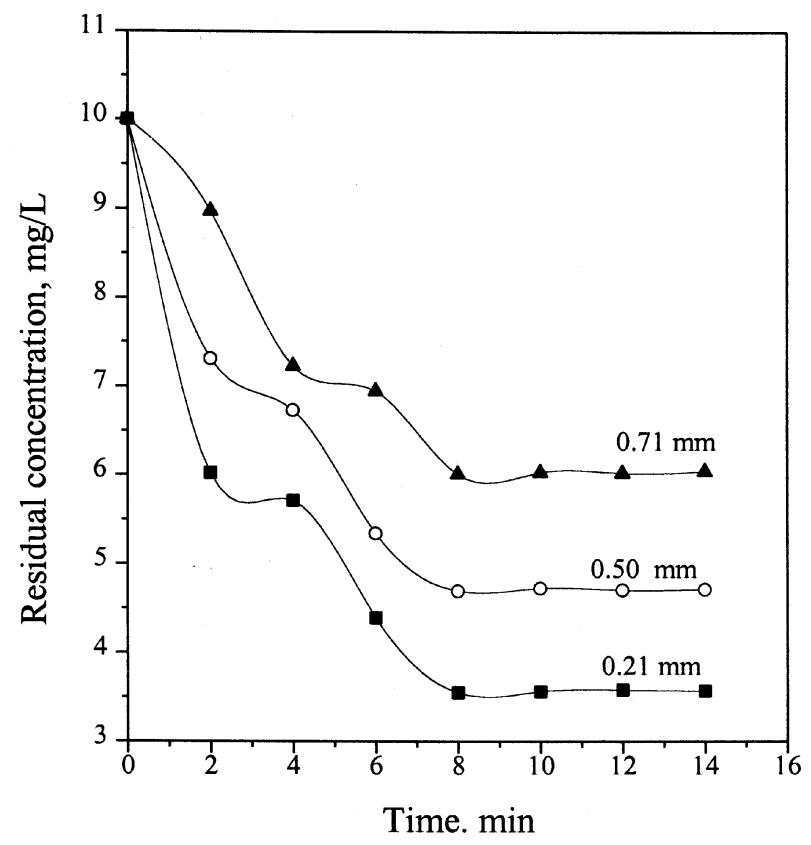

Figure 1. Effect of particle size. 
is a steep increase in the adsorption in the initial stages of the reaction as seen in the curve. ${ }^{6}$ Maximum adsorption occurs at the 8th minute after which the adsorption remains uniform, represented by the attainment of equilibrium. The amount of iron(III) adsorbed at this point is the maximum under the particular operating conditions. The time variation curve is smooth and continuous and this indicates the formation of a monolayer coverage on the outer interface of the adsorbent. $^{7-9}$

\subsection{Effect of initial concentration}

The adsorption curves of iron(III) from solutions containing different initial concentrations are shown in figure 3. The dependence of the system on the concentration factor is explained by low iron(III) to chitin ratio at lower concentrations. Subsequently, the fractional adsorption becomes independent of the initial concentration. ${ }^{10}$ However, at higher concentrations, the available sites of adsorption become fewer and hence the percentage removal of iron(III) is dependent upon the initial concentration. It is evident from the figure that the adsorption rate increased with an increase in the concentration. The actual amount of iron(III) adsorbed is more for higher equilibrium concentration value, $C_{e}$ at increasing concentrations of the adsorbate. ${ }^{11}$ Similar findings

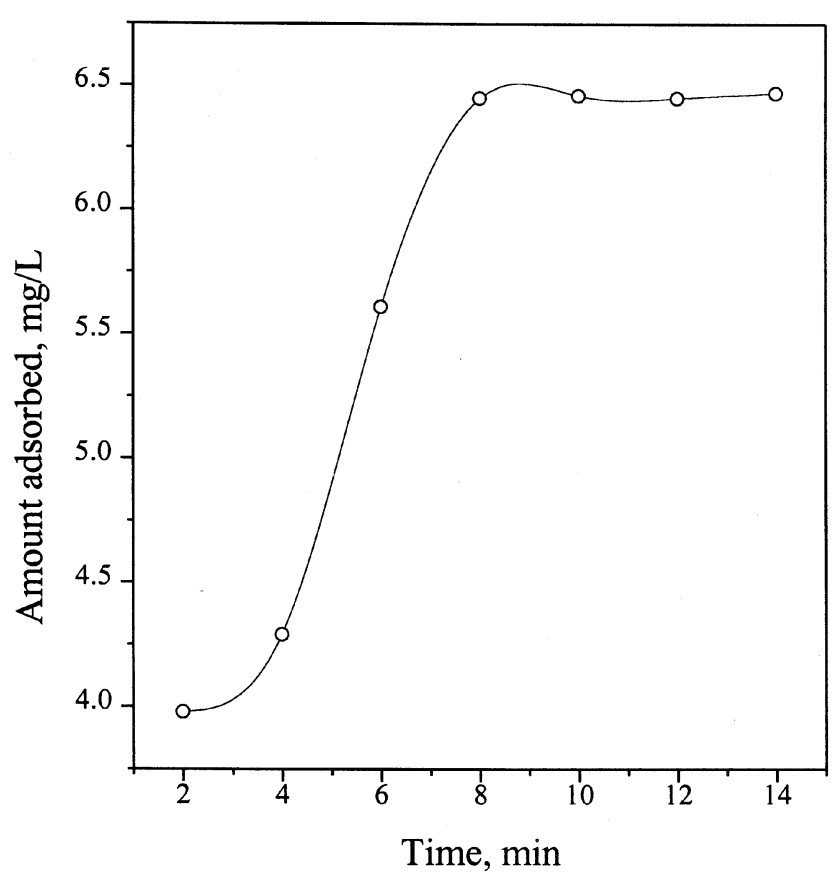

Figure 2. Effect of contact time. were reported by Rao et $a l^{12}$ while working on Blue MR using activated carbon. In the present study the initial concentration of the iron(III) solutions was maintained at $10 \mathrm{mg} / \mathrm{l}$ for all the adsorption experiments.

\subsection{Effect of dosage}

Results of the adsorption experiments using three different dosages, viz. 10, 20 and $30 \mathrm{mg}$ of chitin and $10 \mathrm{mg} / \mathrm{l}$ of iron(III) are given in table 2 . The values indicate an increase in the adsorption capacity of chitin with dosage. This is because of an increase in the number of available adsorption sites of the adsorbent which results in enhanced removal of iron(III). ${ }^{13}$ Rate of adsorption of iron(III) by chitin is apprecia-

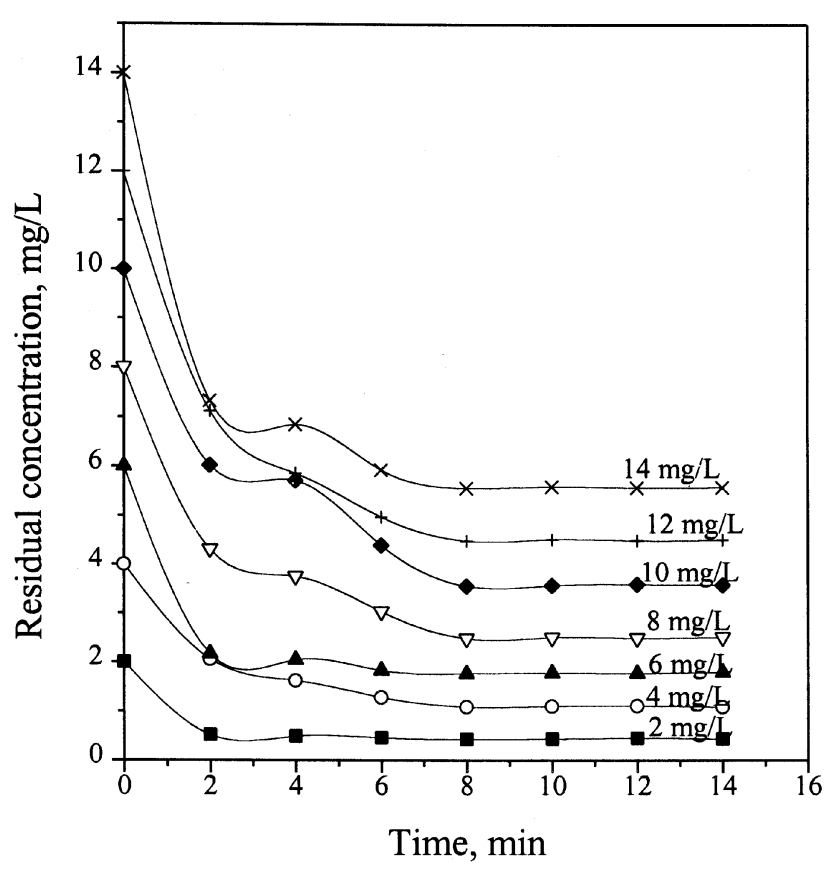

Figure 3. Effect of initial concentration.

Table 1. Effect of contact time.

\begin{tabular}{lc}
\hline Time $(\mathrm{min})$ & Amount adsorbed $(\mathrm{mg} / \mathrm{l})$ \\
\hline 2 & 3.98 \\
4 & 4.29 \\
6 & 5.61 \\
8 & 6.45 \\
10 & 6.46 \\
12 & 6.45 \\
14 & 6.47 \\
\hline
\end{tabular}

Initial concentration of iron(III) $=10 \mathrm{mg} / \mathrm{l} ; \mathrm{pH}=3 \cdot 0$; temperature $=303 \mathrm{~K}$; adsorbant dose $=10 \mathrm{mg}$; particle size $=0.21 \mathrm{~mm}$ 
ble even at an initial dose of $10 \mathrm{mg}$ although at higher doses like 20 and $30 \mathrm{mg}$ it records an increase. Further, the rate of adsorption does not register a proportionate increase as per the experimental results. In view of this observation, it was decided to conveniently fix the dose of chitin as $10 \mathrm{mg}$ for the remaining experiments. Since the particle size and contact time of the adsorbent material, determined as above, accounts for the maximum removal of adsorbate, this was taken as the optimum condition for further adsorption studies using chitin.

Table 2. Effect of dosage.

\begin{tabular}{lccc}
\hline & \multicolumn{3}{c}{$\begin{array}{c}\text { Amount adsorbed (mg/l) at } \\
\text { concentration (in mg) }\end{array}$} \\
\cline { 2 - 4 } Time (min) & 10 & 20 & 30 \\
\hline 2 & 3.98 & 5.69 & $7 \cdot 01$ \\
4 & 4.29 & $6 \cdot 38$ & $7 \cdot 87$ \\
6 & 5.61 & $7 \cdot 11$ & $8 \cdot 11$ \\
8 & 6.45 & 7.85 & 8.73 \\
10 & 6.46 & 7.82 & 8.71 \\
12 & 6.45 & 7.84 & 8.70 \\
14 & 6.47 & 7.83 & 8.72 \\
\hline
\end{tabular}

Initial concentration of iron(III) $=10 \mathrm{mg} / \mathrm{l} ; \mathrm{pH}=3 \cdot 0$; temperature $=303 \mathrm{~K}$; particle size $=0.21 \mathrm{~mm}$

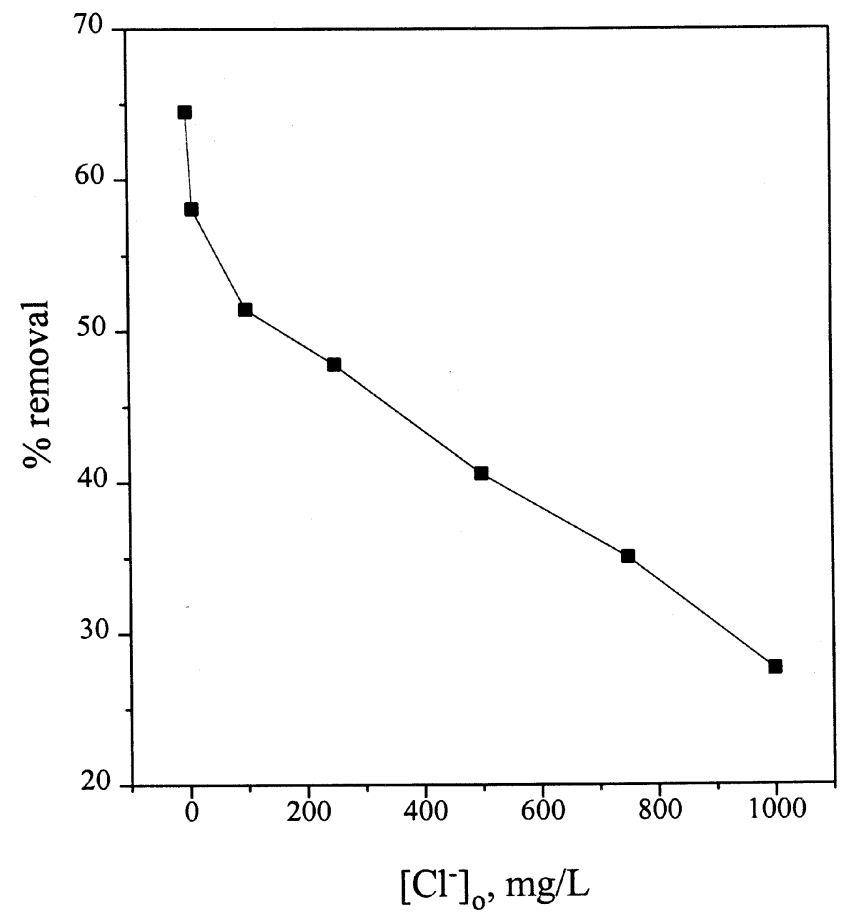

Figure 4. Effect of chloride ion concentration.

\subsection{Effect of chloride, nitrate and sulphate}

Results of the adsorption experiments showing the effects of chloride, nitrate and sulphate ions on the amount of $\mathrm{Fe}$ (III) ions removed by chitin are provided in table 3. The influence of chloride ions on the adsorption of iron(III) by chitin is illustrated in figure 4. The percentage of iron(III) removed decreases from $64.5 \%$ to $27.65 \%$ as the chloride ion concentration increases from $0-1000 \mathrm{mg} / \mathrm{l}$. Formation of soluble chloro complexes at higher concentrations of chloride is responsible for this observed inhibition rate. ${ }^{14}$ This also results in lowering of free iron(III). The observed decrease in the adsorption rate indicates that these chloro complexes have lesser affinity than free metal ions towards chitin. Results indicate similar reduction in the chelating ability of chitin with iron(III) at higher nitrate environments also. This is due to the propensity of iron(III) to complex preferentially with nitrate rather than getting adsorbed onto the chitin surface. ${ }^{15}$ Adsorption studies of iron(III) by chitin in the presence of excess sulphate ions indicate a two-fold decrease in the percentage of iron(III) removed. The results reveal that $42.39 \%$ of iron(III) is reduced in $500 \mathrm{mg} / \mathrm{l}$ of the sulphate environment whereas the amount reduced is $21.43 \%$ at a sulphate concentration of $1000 \mathrm{mg} / \mathrm{l}$.

\subsection{Effect of ionic strength}

Figure 5 shows the influence of ionic strength on the adsorption of iron(III) by chitin. Perchlorate solu-

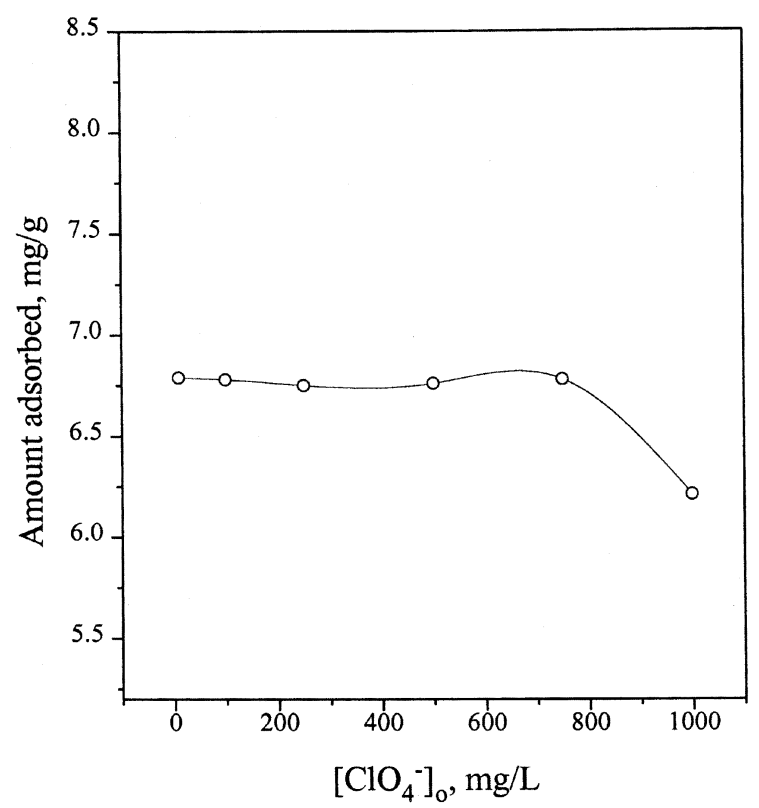

Figure 5. Effect of ionic strength. 
Table 3. Effect of chloride, nitrate and sulphate.

\begin{tabular}{lccccc}
\hline$\left[\mathrm{Cl}^{-}\right]_{0}$ & \% removal & {$\left[\mathrm{NO}_{3}^{-}\right]_{0}$} & $\%$ removal & {$\left[\mathrm{SO}_{4}^{2-}\right]_{0}$} & $\%$ removal \\
\hline 0 & $64 \cdot 50$ & 0 & $64 \cdot 50$ & 0 & $64 \cdot 50$ \\
10 & $58 \cdot 09$ & 10 & $46 \cdot 63$ & 10 & $58 \cdot 80$ \\
100 & 51.46 & 100 & 41.52 & 100 & $51 \cdot 22$ \\
250 & $47 \cdot 78$ & 250 & $38 \cdot 56$ & 250 & $45 \cdot 33$ \\
500 & 40.56 & 500 & $36 \cdot 42$ & 500 & $42 \cdot 39$ \\
750 & 35.03 & 750 & $35 \cdot 94$ & 750 & $35 \cdot 59$ \\
1000 & 26.65 & 1000 & $32 \cdot 48$ & 1000 & $21 \cdot 43$ \\
\hline
\end{tabular}

Initial concentration of iron(III) $=10 \mathrm{mg} / \mathrm{l} ; \mathrm{pH}=3.0$; temperature $=303 \mathrm{~K}$; adsorbant dose $=10 \mathrm{mg}$; contact time $=8 \mathrm{~min}$; particle size $=0.21 \mathrm{~mm}$

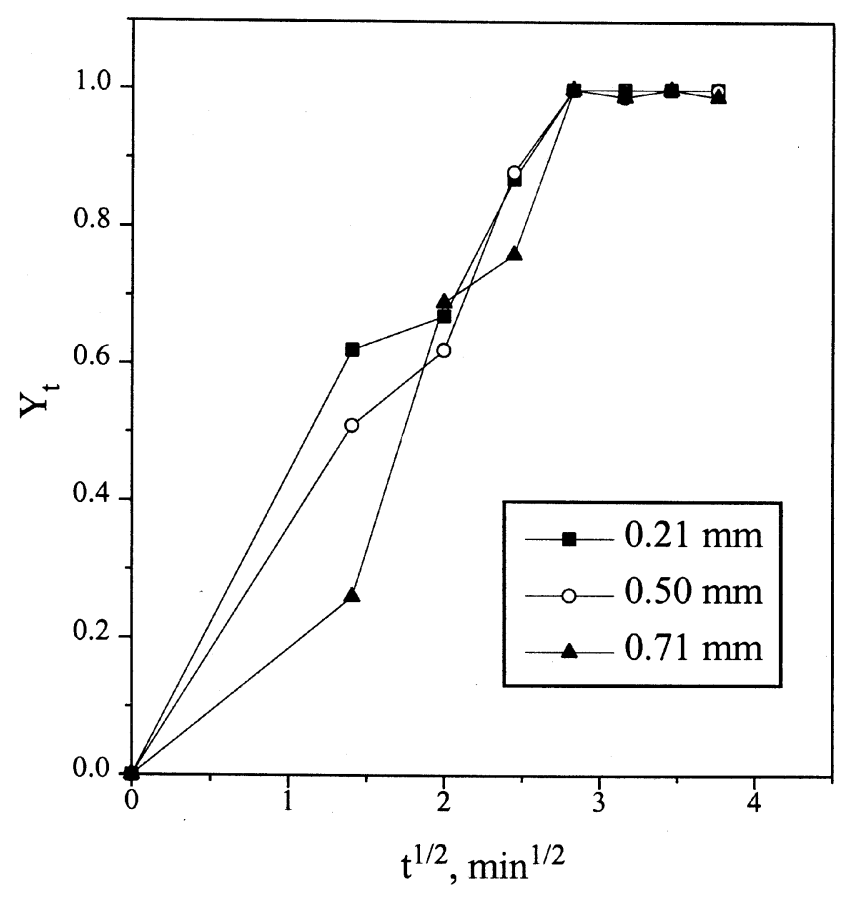

Figure 6. Time dependence of the fraction of adsorption at different particle sizes of chitin.

tions ranging from $10-1000 \mathrm{mg} / \mathrm{l}$ were prepared and mixed with ferric solution of $10 \mathrm{mg} / \mathrm{l}$ concentration. The absence of any significant decrease in the uptake of iron(III) in the presence of sodium perchlolrate up to $750 \mathrm{mg} / \mathrm{l}$ is evident from the absence of any curve as indicated in the figure. However, at $1000 \mathrm{mg} / \mathrm{l}$ of perchlorate concentration, considerable reduction in the amounts of iron(III) adsorbed is evident, as implied by the bend in the curve. In other words, the increased amount of perchlorate $(1000 \mathrm{mg} / \mathrm{l})$ helps to swamp the surface of chitin, which decreases the access of $\mathrm{Fe}(\mathrm{III})$ ions to the chitin surface for adsorption. Adsorption is sensitive to changes in the concentration of the supporting electrolytes, if electrostatic at- traction is the significant mechanism for sorbate removal. It is therefore concluded that electrostatic attraction plays a negligible role in the removal of iron(III) by chitin. ${ }^{16}$

\subsection{Time-dependence of fraction of adsorption at different particle sizes and doses of chitin}

Mechanism of sorption of iron(III) by chitin is explained by the fraction of adsorption $Y_{t}$. The plots of $Y_{t}$ (figures 6 and 7) for different particle sizes and dosages have three-stage shapes indicating external surface adsorption, intraparticle diffusion and final equilibrium. The values given in tables 4 and 5 show enhanced rates of sorption at increased dosage levels and reduced particle sizes. The first, sharp portion of the curve corresponds to the external surface adsorption stage or instantaneous adsorption stage. The second, less-sloping linear portion indicates the gradual adsorption stage and the final portion represents the equilibrium stage. In this model, the chitin sample is treated as being surrounded by a boundary layer film through which the metal ions must diffuse prior to adsorption on the chitin surface. The second portion of the figures indicates the intraparticle diffusion, which is predominant in the rate-controlling step where diffusive transport occurs through the internal pores of the adsorbent. As the bulk and surface metal concentrations start to decrease, the third section of the figures shows decrease in the rate of diffusion reaching a point of equilibrium. ${ }^{17}$ Both increasing dose and decreasing particle size increase the driving force of metal ions from the bulk phase on and into the solid chitin.

An examination of the values of $t^{1 / 2}$ and $Y_{t}$ leads to the following facts. The fraction of adsorption in terms of retention time, $t^{1 / 2}$ shows that the first stage corresponds to a retention time of $1.41 \mathrm{~min}^{1 / 2}$. The 
second stage corresponds to a retention time for $t^{1 / 2}$ between $1.41 \mathrm{~min}^{1 / 2}$ and $2.45 \mathrm{~min}^{1 / 2}$. Values of $t^{1 / 2}$, above $2.45 \mathrm{~min}^{1 / 2}$ represent the equilibrium stage reached and this corresponds to the third stage.

\subsection{Intraparticle diffusion}

Plots of time squared and amount adsorbed for particle sizes and dosages of chitin as variables are shown in figures 8 and 9 . The initial curved portions represent the boundary-layer diffusion effect. The intraparticle diffusion rate constants, $k_{p}$, calculated from the slopes of the linear portion of curves which represent the intraparticle diffusion effects are given in tables 6 and 7. ${ }^{7,18}$ The $k p$ values increase with decrease in particle

Table 4. Time dependence of the fraction of adsorption at different particle sizes of chitin.

\begin{tabular}{lccc}
\hline & \multicolumn{3}{c}{$Y_{t}(\mathrm{~mm})$} \\
\cline { 2 - 4 }$t^{1 / 2}\left(\mathrm{~min}^{1 / 2}\right)$ & 0.21 & 0.50 & 0.71 \\
\hline 0 & 0 & 0 & 0 \\
1.41 & 0.62 & 0.51 & 0.26 \\
2.00 & 0.67 & 0.62 & 0.69 \\
2.45 & 0.87 & 0.88 & 0.76 \\
2.83 & 1.00 & 1.00 & 1.00 \\
3.16 & 1.00 & 0.99 & 0.99 \\
3.46 & 1.00 & 1.00 & 1.00 \\
3.76 & 1.00 & 1.00 & 0.99 \\
\hline
\end{tabular}

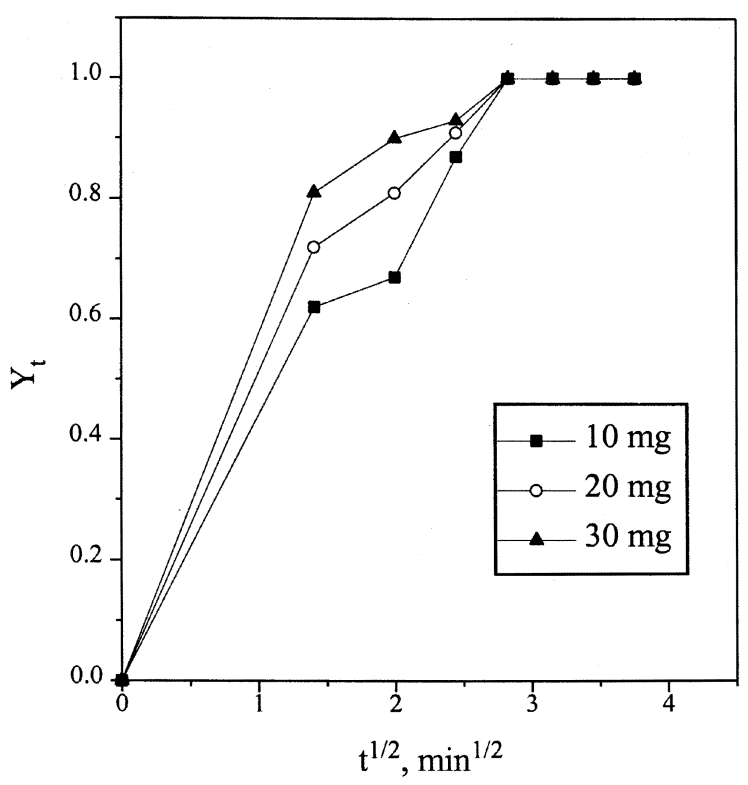

Figure 7. Time dependence of the fraction of adsorption at different doses of chitin. size and increase in dosage levels of chitin. Otherwise, increasing the particle size of the adsorbent requires more time to reach equilibrium. This results in the lowering of intraparticle diffusion rate constants at larger particle sizes and vise versa smaller particles sizes. Values of $k_{p}$ increase with the dosage of chitin, due to increase in the number of chitin molecules as more active binding sites are available for iron(III) to diffuse into.

\subsection{Effect of co-ions}

Experimental results of the co-ionic effect on the adsorption of iron(III) ions from an aqueous solution containing copper(II), zinc(II) and chromium(VI) in

Table 5. Time dependence of the fraction of adsorption at different doses of chitin.

\begin{tabular}{lccc}
\hline & \multicolumn{3}{c}{$Y_{t}(\mathrm{mg})$} \\
\cline { 2 - 4 }$t^{1 / 2}\left(\mathrm{~min}^{1 / 2}\right)$ & 10 & 20 & 30 \\
\hline 0 & 0 & 0 & 0 \\
1.41 & $0 \cdot 62$ & $0 \cdot 72$ & $0 \cdot 81$ \\
2.00 & 0.67 & 0.81 & $0 \cdot 90$ \\
2.45 & 0.87 & 0.91 & 0.93 \\
2.83 & 1.00 & 1.00 & 1.00 \\
3.16 & 1.00 & 1.00 & 1.00 \\
3.46 & 1.00 & 1.00 & 1.00 \\
3.76 & 1.00 & 1.00 & 1.00 \\
\hline
\end{tabular}

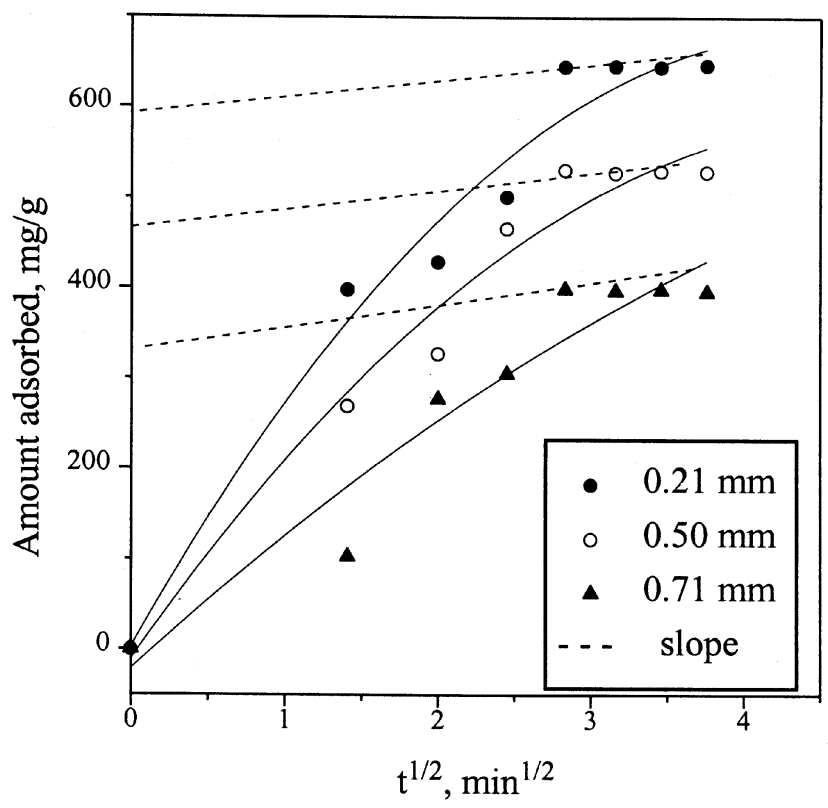

Figure 8. Intraparticle diffusion plot for the adsorption of $\mathrm{Fe}(\mathrm{III})$ on chitin at different particle sizes. 
equal concentrations reveal that the presence of foreign ions diminishes the adsorption of iron(III) ions. However, the presence of an equal amount of copper(II) ions reduces the adsorption rate considerably,

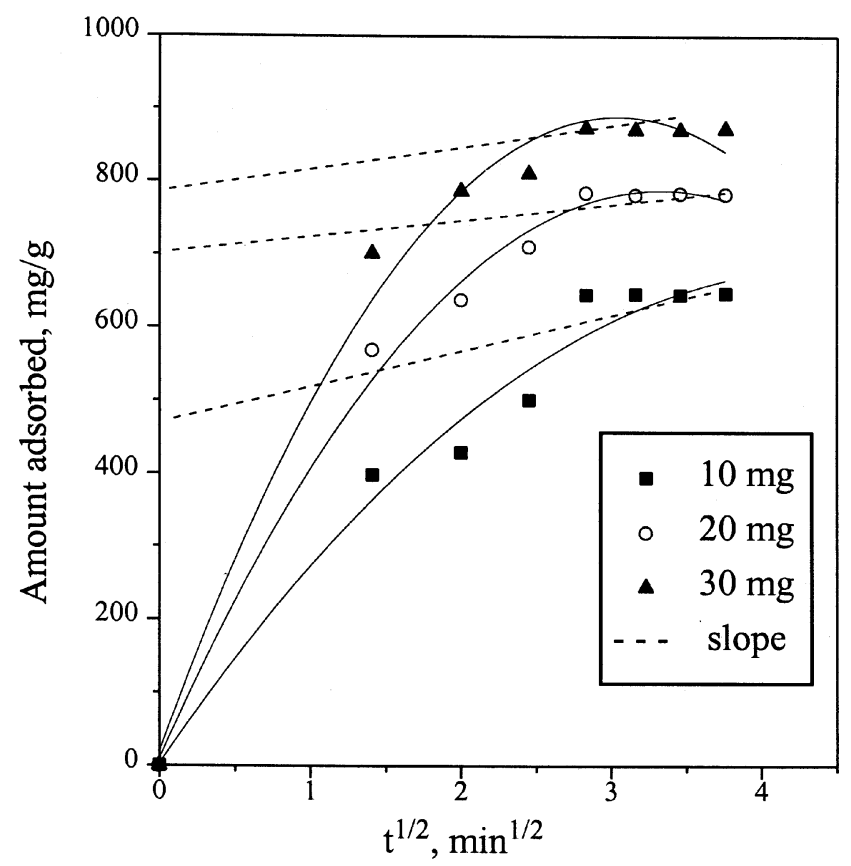

Figure 9. Intraparticle diffusion plot for the adsorption of $\mathrm{Fe}(\mathrm{III})$ on chitin at different doses.

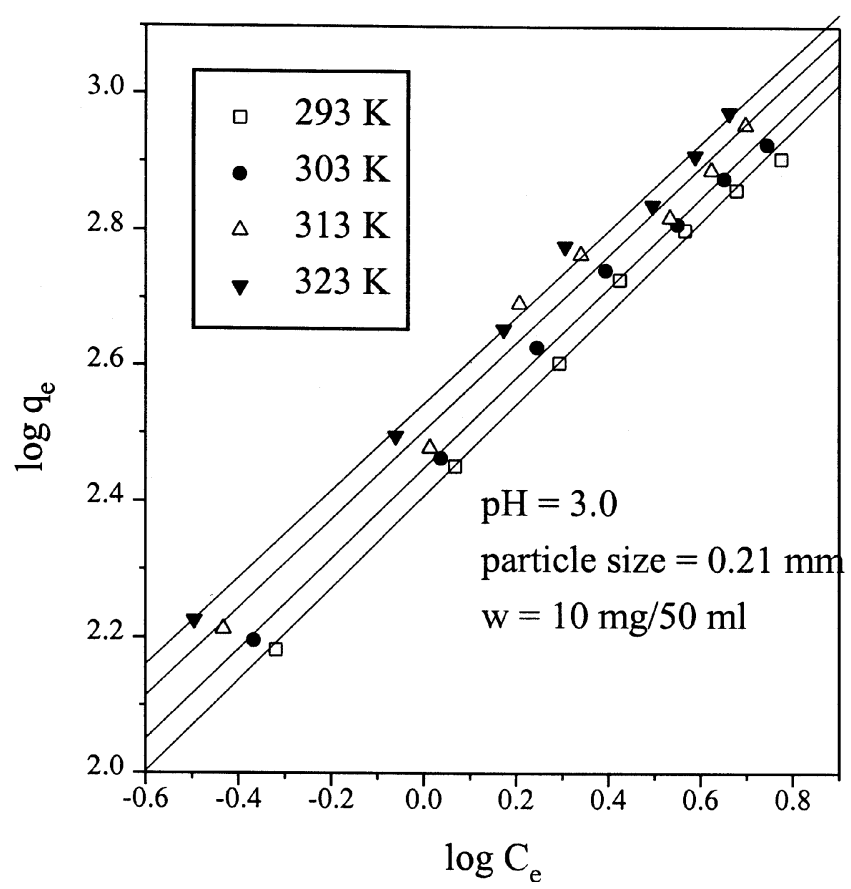

Figure 10. Freundlich isotherm. while the presence of chromium(VI) and zinc(II), has a lesser effect. Jha et $a l^{16}$ have reported similar co-ionic effects in the removal of specific metal ions in their studies.

Table 6. Intraparticle diffusion rate constants depending on particle size

\begin{tabular}{llll}
\hline Particle size $(\mathrm{mm})$ & 0.21 & 0.50 & 0.71 \\
$k_{p}$ & 2.09 & 1.67 & 1.51
\end{tabular}

Table 7. Intraparticle diffusion rate constants depending on dosage.

\begin{tabular}{lccc}
\hline Dosage $(\mathrm{mg})$ & 10 & 20 & 30 \\
$k_{p}$ & $2 \cdot 09$ & 2.61 & 3.23 \\
\hline
\end{tabular}

Table 8. Freundlich parameters.

\begin{tabular}{lcc}
\hline Temperature $(\mathrm{K})$ & $K$ & $1 / n$ \\
\hline 293 & $2 \cdot 41$ & 0.68 \\
303 & $2 \cdot 45$ & 0.67 \\
313 & $2 \cdot 50$ & 0.65 \\
323 & 2.54 & 0.64 \\
\hline
\end{tabular}

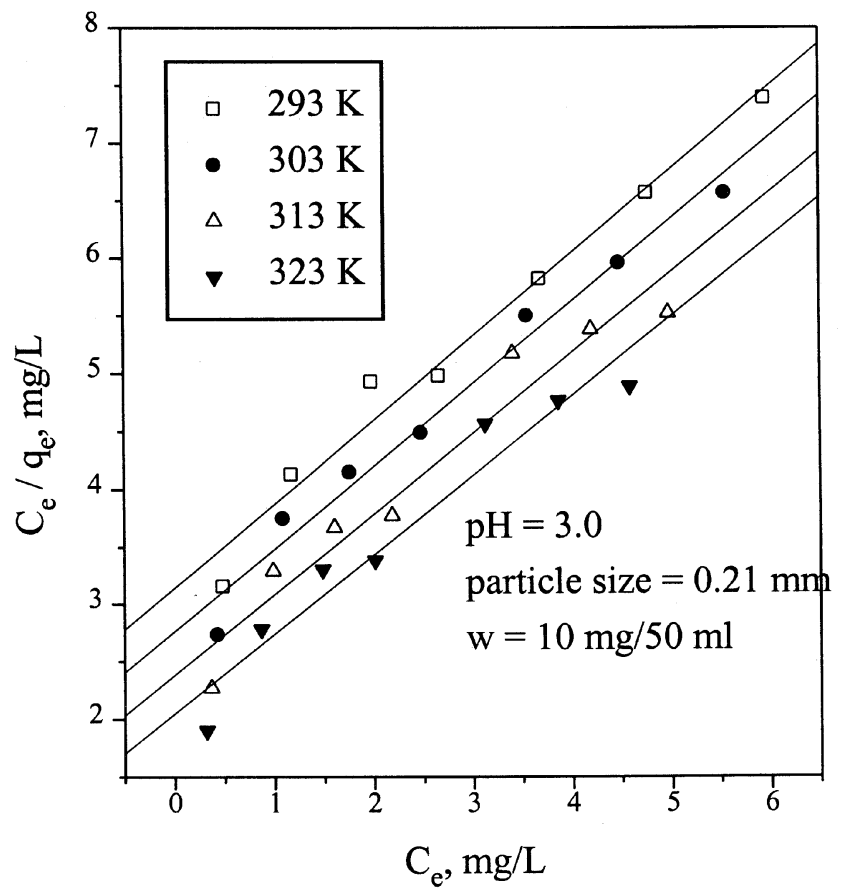

Figure 11. Langmuir isotherm. 
Table 9. Effect of temperature on equilibrium parameters.

\begin{tabular}{|c|c|c|c|c|c|c|c|c|}
\hline \multirow{2}{*}{$\begin{array}{l}\text { Concentration } \\
\text { of } \mathrm{Fe}(\mathrm{III})(\mathrm{mg} / \mathrm{l})\end{array}$} & \multicolumn{4}{|c|}{$C_{e}(\mathrm{mg} / \mathrm{l})$ at temp. $(\mathrm{K})$} & \multicolumn{4}{|c|}{$C_{e} / q_{e}(\mathrm{mg} / \mathrm{l})$ at temp. $(\mathrm{K})$} \\
\hline & 293 & 303 & 313 & 323 & 293 & 303 & 313 & 323 \\
\hline 2 & 0.48 & 0.43 & $0 \cdot 37$ & 0.32 & $3 \cdot 16$ & $2 \cdot 74$ & $2 \cdot 27$ & 1.90 \\
\hline 4 & $1 \cdot 17$ & 1.09 & 0.99 & 0.87 & $4 \cdot 13$ & 3.75 & $3 \cdot 29$ & 2.78 \\
\hline 6 & 1.98 & 1.76 & 1.61 & 1.49 & 4.93 & $4 \cdot 15$ & 3.67 & $3 \cdot 30$ \\
\hline 8 & $2 \cdot 66$ & 2.48 & $2 \cdot 19$ & 2.02 & 4.98 & 4.49 & 3.77 & $3 \cdot 38$ \\
\hline 10 & 3.68 & 3.55 & 3.41 & $3 \cdot 13$ & 5.82 & 5.50 & $5 \cdot 17$ & 4.56 \\
\hline 12 & $4 \cdot 76$ & 4.48 & $4 \cdot 20$ & 3.87 & 6.57 & 5.96 & $5 \cdot 38$ & 4.76 \\
\hline 14 & 5.95 & 5.55 & 4.98 & 4.59 & $7 \cdot 39$ & 6.57 & $5 \cdot 52$ & $4 \cdot 88$ \\
\hline
\end{tabular}

Table 10. Langmuir constants and thermodynamic parameters.

\begin{tabular}{|c|c|c|c|c|c|c|}
\hline \multirow[b]{2}{*}{ Temp. (K) } & \multicolumn{2}{|c|}{ Langmuir constants } & \multicolumn{4}{|c|}{ Thermodynamic parameters } \\
\hline & $b\left(1 \mathrm{mg}^{-1}\right)$ & $Q\left(\mathrm{mg} \mathrm{g}^{-1}\right)$ & $K_{o}$ & $G^{\mathrm{o}}\left(\mathrm{kJ} \mathrm{mol}^{-1}\right)$ & $H^{\mathrm{o}}\left(\mathrm{kJ} \mathrm{mol}^{-1}\right)$ & $S^{\mathrm{o}}\left(\mathrm{J} \mathrm{mol}^{-1} \mathrm{~K}^{-1}\right)$ \\
\hline 293 & 0.2311 & $1 \cdot 3778$ & $5.88 \pm 0.06$ & $-4.32 \pm 0.03$ & $2 \cdot 16$ & $22 \cdot 06$ \\
\hline 303 & 0.2591 & $1 \cdot 3982$ & $6 \cdot 02 \pm 0.06$ & $-4.52 \pm 0.03$ & & \\
\hline 313 & 0.2934 & 1.4323 & $6 \cdot 17 \pm 0 \cdot 11$ & $-4 \cdot 72 \pm 0 \cdot 04$ & & \\
\hline 323 & 0.3356 & 1.4537 & $6 \cdot 36 \pm 0 \cdot 10$ & $-4.97 \pm 0.04$ & & \\
\hline
\end{tabular}

\subsection{Adsorption isotherms}

Freundlich and Langmuir adsorption isotherm models were applied for adsorption equilibrium at all four temperatures.

3.10a Freundlich isotherm: Batch isothermal data fitted to the linear form of the Freundlich isotherm are shown in figure 10. The isothermal equation is as follows:

$\log q_{e}=\log K+1 / n \log C_{e}$,

where $q_{e}$ is the amount of copper sorbed per unit weight of the sorbent $(\mathrm{mg} / \mathrm{g})$ and $C_{e}$ is the equilibrium concentration $(\mathrm{mg} / \mathrm{l})$ of $\mathrm{Fe}(\mathrm{III})$ in solution. The fit of the data imply that the intercept, $K$, is roughly an indicator of the sorption capacity and the slope, $1 / n$, of the sorption intensity, the constants incorporating all the factors affecting the adsorption process. Values of $K$ and $1 / n$ are calculated from the graph and the data are provided in table 8 . The linearity of the plots $(0.9911<r<0.9979$ and $0.0185<\mathrm{sd}<$ 0.0378 ) at different temperatures is evident from figure 10. This supports the applicability of the Freundlich adsorption isotherm indicating that the adsorption by chitin may be governed by physisorption. ${ }^{19}$ The values of $n$ for this system were calculated from the slope of the curve and are found to be between 1 and 2. Our findings are in good agreement with the findings of Treybal $^{20}$ who mathematically evaluated values of $n$ for a number of mass transfer operations of systems and reported that values of $n$ between 1 and 10 would represent beneficial adsorption.

3.10b Langmuir isotherm: The linear equation of the Langmuir adsorption isotherm was applied in the form,

$$
C_{e} / q_{e}=1 / Q b+C_{e} / Q,
$$

where $Q$ and $b$ are the Langmuir constants, indicating adsorption capacity and energy of adsorption respectively. $C_{e}$ and $q_{e}$ have their usual significance. The values for the plot $C_{e} / q_{e}$ versus $C_{e}$ for the adsorption by chitin at different temperatures are calculated and presented in table 9. The linear plots $(0.9702<$ $r<0.9893$ and $0.2175<s d<0.3212)$ of $C_{e} / q_{e}$ versus $C_{e}$ at different temperatures are given in figure 11 and they indicate the applicability of the Langmuir adsorption isotherm to the adsorption process. The linear form of the regression equations are given below.

$$
\begin{aligned}
& C_{e} / q_{e}=3 \cdot 14 \pm 0.17+0.73 \pm 0.05 C_{e}(\text { at } 293 \mathrm{~K}), \\
& C_{e} / q_{e}=2.76 \pm 0.16+0.72 \pm 0.05 C_{e}(\text { at } 303 \mathrm{~K}), \\
& C_{e} / q_{e}=2.38 \pm 0.23+0.70 \pm 0.08 C_{e}(\text { at } 313 \mathrm{~K}),
\end{aligned}
$$


$C_{e} / q_{e}=2 \cdot 05 \pm 0 \cdot 21+0.69 \pm 0.08 C_{e}($ at $323 \mathrm{~K})$.

Values of $Q$ and $b$ at different temperatures were determined from the above plots and are given in table 10 . An increase in $K_{o}$ value with temperature indicates the endothermic character of the adsorption. The re-

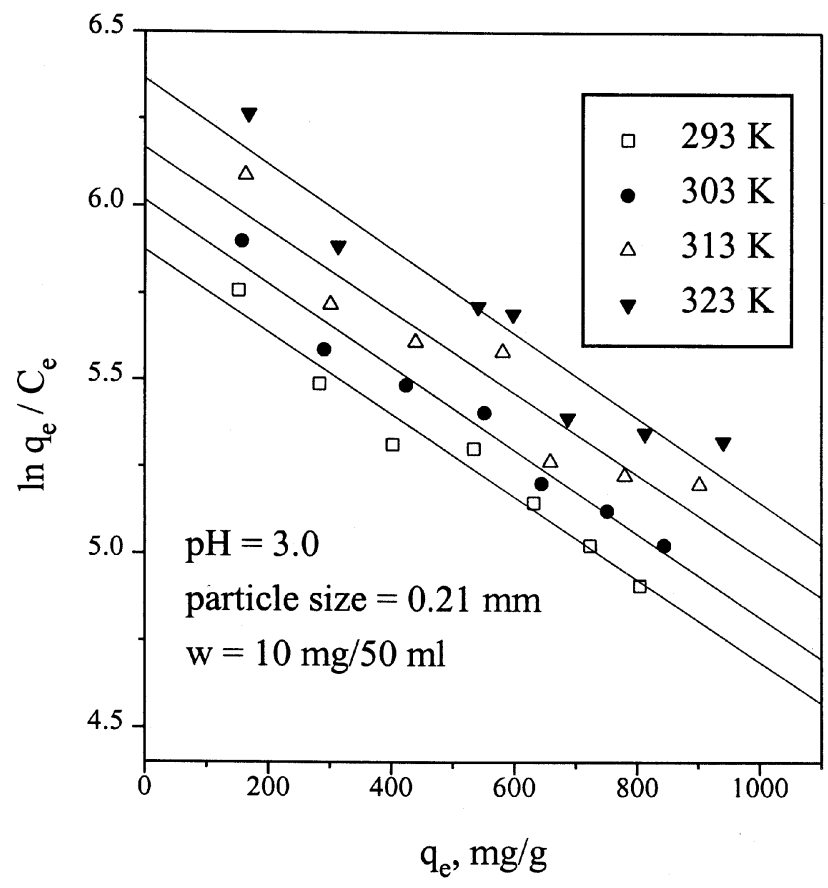

Figure 12. Plot of $\ln q_{e} / C_{e}$ versus $q_{e}$.

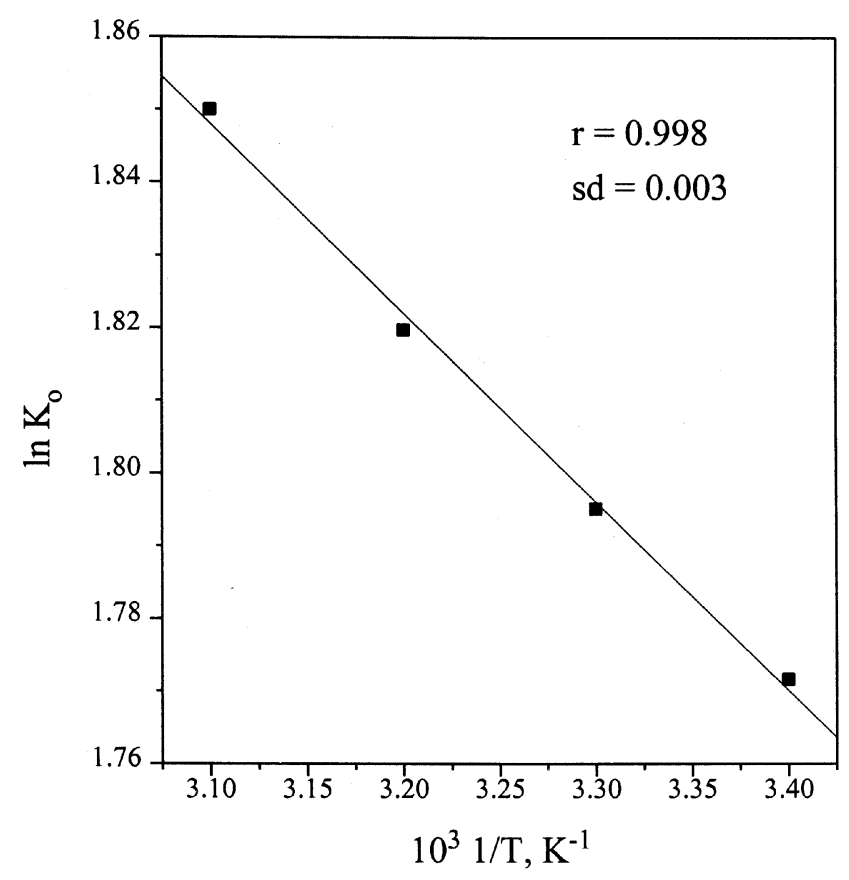

Figure 13. Plot of $\ln K_{0}$ versus $1 / T$. sults reveal that the energy of adsorption, $b$, for trapping of iron(III) by chitin increases with temperature and hence leads to greater of $\mathrm{Fe}(\mathrm{III})$ adsorption at higher temperatures. ${ }^{21}$ Annadurai et al. ${ }^{22}$ who studied the sorptive character of chitin on basic dyes have also reported similar observations. The influence of temperature on chitin and its enhanced adsorption behaviour further emphasises to the phenomenon of swelling in the internal structure of the chitin molecules to accommodate more iron(III) on its surface.

\subsection{Equilibrium parameter}

Values of the dimensionless equilibrium parameter, $R_{L}$, of different temperatures are calculated for the initial concentrations of iron(III) and are given in table 11. The parameters explain the differences in the shapes of the isotherm. The $R_{L}$ values lying between 0 and 1 indicate favourable adsorption at the four temperatures. ${ }^{7}$ The observed $R_{L}$ values for iron(III) in the solution are 0.18-0.68 and correspond to the concentration range $2-14 \mathrm{mg} / \mathrm{l}$, indicative of the favourable adsorption.

The plots of $\ln q_{e} / C_{e}$ versus $q_{e}$ of the reactions are given in figure 12 . The equilibrium constant, $K_{o}$ for the sorption reaction was determined from the plot $\ln q_{e} / C_{e}$ versus $q_{e}$ and extrapolating to zero $q_{e}$. An increase in $K_{o}$ value with temperature indicates that the adsorption process of iron(III) by chitin is endothermic. The effect of increase in temperature on the adsorption of iron(III) at the chitin-solution interface has been studied in the temperature range of $293-313 \mathrm{~K}$. A plot of $\ln K_{o}$ vs $1 / T$ is found to be linear as shown in figure 13. $\Delta H^{\mathrm{o}}$ and $\Delta S^{\mathrm{o}}$ are determined from the slopes and intercepts of the plot ${ }^{23}$. The negative values of the standard free energy change $\left(\Delta G^{0}\right)$ for this reaction suggests that the sorptive nature is spontaneous. The positive value of $\Delta H^{\mathrm{o}}(2 \cdot 16 \mathrm{~kJ} /$

Table 11. Equilibrium parameter $\left(R_{L}\right)$.

\begin{tabular}{|c|c|c|c|c|}
\hline \multirow{2}{*}{$\begin{array}{l}\text { Concentration } \\
\text { of } \mathrm{Fe}(\mathrm{III})(\mathrm{mg} / \mathrm{l})\end{array}$} & \multicolumn{4}{|c|}{ Temperature $(\mathrm{K})$} \\
\hline & 293 & 303 & 313 & 323 \\
\hline 2 & 0.68 & 0.66 & 0.63 & 0.60 \\
\hline 4 & 0.52 & 0.49 & 0.46 & 0.43 \\
\hline 6 & 0.42 & $0 \cdot 39$ & 0.36 & $0 \cdot 33$ \\
\hline 8 & 0.35 & 0.33 & 0.30 & 0.27 \\
\hline 10 & $0 \cdot 30$ & $0 \cdot 28$ & 0.25 & 0.23 \\
\hline 12 & 0.27 & $0 \cdot 24$ & 0.22 & 0.20 \\
\hline 14 & 0.24 & 0.22 & $0 \cdot 20$ & 0.18 \\
\hline
\end{tabular}


mo1) indicates that the process of adsorption is endothermic and irreversible, probably due to nonpolar interactions. ${ }^{24}$ The positive value of entropy change $\Delta S^{\mathrm{o}}(22.06 \mathrm{~J} / \mathrm{K} / \mathrm{mo} 1)$ suggests a high degree of disorderliness at the solid-solution interface during the adsorption process of iron(III) by chitin. ${ }^{25}$ It also reflects the affinity of the adsorbent for iron(III) and suggests some structural changes in adsorbate and adsorbent. Enhancement of adsorption capacity of chitin at higher temperatures may be attributed to the enlargement of the pore size and/or activation of the adsorbent surface and increase in the mobility of the metal ions.

\section{Conclusion}

Based on the experimental results, it is observed that the adsorption of $\mathrm{Fe}^{3+}$ ions by chitin is maximum with the least particle size and minimum contact time of eight minutes. Sorption of $\mathrm{Fe}^{3+}$ ions increases at higher initial concentrations and doses. The influence of $\mathrm{pH}$ on the adsorption of iron(III) by chitin could not be studied as its solution itself is acidic. The inhibitive effects of chloride, nitrate and sulphate and the absence of any significant influence of ionic strength over the sorption rate are observed. However, marked reduction in the sorption process is noticed under the influence of co-ions. $Y_{t}$ plots depict the three stages in the sorption process; the calculated $k_{p}$ values increase with dosages and decrease with particle sizes. The Fe(III)-chitin system follows Freundlich and Langmuir isotherms which indicates the governance of physisorption and/or chemisorption. Values of thermodynamic functions show that the reaction is endothermic, random and beneficial.

\section{References}

1. Arms K 1990 Environmental science (San Francisco, CA: Saunders College)
2. Lauffer R 1992 Iron and human diseases (London: CRC Press)

3. Emery T 1991 Iron and your health: Facts and fallacies (London: CRC Press)

4. Gonzalez-Davila M, Santana-Casiano M J and Millero F J 1990 J. Colloid. Inter. Sci. 137102

5. Slejko F 1985 Adsorption technology: A step by step approach to process calculation and application (New York: Marcel Decker)

6. Singh D K and Bhavna Srivastava 2001 Indian J. Chem. Technol. 8133

7. Singh D K, Garg S K and Bharadwaj R K 2001 Indian J. Environ. Protection 21604

8. Khattri S D and Singh M K 1999 Indian J. Chem. Technol. 6112

9. Desai M, Dogra A, Vora S, Bahadur P and Ram R N 1997 Indian J. Chem. A36 938

10. Parekh D C, Patel J B, Sudhakar P and Koshy V J 2002 Indian J. Chem. Technol. 9540

11. De D S and Basu J K 1999 Indian J. Environ. Protection 19416

12. Rao L N, Krishnaiah K C K and Ashutosh A 1994 Indian J. Chem. Technol. 113

13. Siddiqui B A, Sharama P P and Sultan M 1999 Indian J. Environ. Protection 19846

14. Anirudhan T S and Sreedhar M K 1998 Poll. Res. 17 381

15. Viswanadham M, Sriramulu N and Chary A M 2000 Indian J. Environ. Protection 20515

16. Jha I N, Iyengar L and Prabhakara Rao A V S 1998 J. Environ. Engg. 114962

17. Ruey-Shin Juang, Ru-Ling Tseng, Feng-Chin Wu, Shwu-Hwa Lee 1997 J. Chem. Tech. Biotechnol. 70391

18. Rengaraj S, Sivabalan R, Arabindo B and Murugesan V 2000 J. Indian Chem. Soc. 7127

19. Rama Mohan K, Ramesh A and Seshaiah K 2001 Indian J. Chem. Technol. 8278

20. Treybal R E 1980 Mass transfer operations (New York: McGraw Hill)

21. Karthikeyan G, Pius A and Alagumuthu G 2002 Indian J. Chem. Technol. 9397

22. Annadurai G, Chellapandian M and Krishnan M R V 1997 Indian J. Environ. Protection 1795

23. Manju G N, Gigi M C and Anirudhan T S 1999 Indian J. Chem. Technol. 6134

24. Stephan Inbaraj B and Sulochana N 2002 Indian J. Chem. Technol. 9201

25. Viswakarma P P, Yadava K P and Singh V N 1989 Pertanika 12257 